\title{
A Case of Expansion of Traumatic Choroidal Rupture with Delayed-Developed Outer Retinal Changes
}

\author{
Kun Moon ${ }^{a} \quad K_{w a n g}$ Soo Kim ${ }^{b} \quad$ Yu Cheol Kim ${ }^{b}$ \\ ${ }^{a}$ Department of Ophthalmology, Samsung Changwon Hospital, Sungkyunkwan University \\ School of Medicine, Changwon, and ${ }^{b}$ Department of Ophthalmology, Dongsan Medical \\ Center, Keimyung University School of Medicine, Daegu, South Korea
}

\section{Key Words}

Choroid $\cdot$ Rupture $\cdot$ Injuries

\begin{abstract}
Background: This study aims to report the expansion of a choroidal rupture site caused by blunt ocular trauma using scanning laser ophthalmoscope-optical coherence tomography. Case Report: The clinical course of a 15-year-old girl with a traumatic ocular injury after being hit in the eye by an elbow was evaluated. Upon the first examination, the bestcorrected visual acuity was 16/20. The findings were subretinal pigment epithelial hemorrhage and vertical choroidal rupture. Three weeks after the trauma, the patient's visual acuity was reduced to counting fingers at $30 \mathrm{~cm}$. On fundus examination and fluorescein angiography subretinal pigment epithelial hemorrhage decreased, while the choroidal rupture expanded; on optical coherence tomography outer retinal changes in the adjacent area were detected. Discussion: This study reveals that the delayed expansion of a choroidal rupture site and the delayed change of the adjacent outer retina improved.
\end{abstract}

\section{Introduction}

Choroidal rupture is an uncommon complication of blunt ocular trauma; it was first described in 1854 by Von Graefe as a tear of the choroid, Bruch's membrane, and the retinal pigment epithelium (RPE) [1]. Choroidal rupture can occur directly at the site of impact or 
Moon et al:: A Case of Expansion of Traumatic Choroidal Rupture with DelayedDeveloped Outer Retinal Changes

indirectly on the opposite globe side by a countercoup injury; the latter variation makes up $80 \%$ of all cases. Indirect choroidal rupture is seen on the temporal side of the optic nerve, and when the macula is involved, a significant deleterious effect to the vision results. Indirect choroidal rupture develops in approximately $5 \%$ of patients after blunt ocular injuries [2]. And $5-20 \%$ of eyes develop secondary choroidal neovascularization (CNV) after the rupture heals [3-5].

This case is being presented since it shows a vertical indirect choroidal rupture in the macula and an expansion of the choroidal rupture site that is accompanied by changes to the outer retina in the third week after injury. To the best of our knowledge, such as case has not been previously reported.

\section{Case Report}

A 15-year-old girl presented with blurred vision after being hit by an elbow on her left eye. Her best-corrected visual acuity (BCVA) was 16/20. On fundus examination, both a choroidal rupture that formed a crescent shape concentric to the optic disc and a subretinal hemorrhage were detected (fig. 1a). Optical coherence tomography (OCT) showed that the juxtafoveal retinal pigment epithelial layer with Bruch's membrane was disrupted, and the inner segment/outer segment (IS/OS) of the photoreceptor junction and the external limiting membrane (ELM) layer were distorted with suspected pinpoint disruption (fig. 2a).

After 3 weeks, her visual acuity reduced to counting fingers. Fundus examination revealed that the subretinal hemorrhage had absorbed and the choroidal rupture had expanded. The crescent-shaped window defect including the fovea was observed on fluorescein angiography (FAG) (fig. 1b). Wider disrupted layers of the RPE, the IS/OS junction, and the ELM at the corresponding area were detected in OCT. When compared with the first medical examination after the injury, the IS/OS junction and the RPE adjacent to the ruptured site were hardly distinguishable, although they were originally clearly observed (fig. 2b, c).

Steroid pulse therapy (methylprednisolone $500 \mathrm{mg}$ intravenous for 3 days and prednisolone $30 \mathrm{mg}$ oral, tapering for 11 days) was started in another hospital, and 3 months after the injury, visual acuity increased to 20/125. Five months later, OCT revealed that the size of the expanded choroidal rupture had reduced, and the adjacent retina had improved sufficiently to distinguish the IS/OS junction and the RPE. However, FAG disclosed a choroidal neovascular membrane originating from the nasal edge of the choroidal rupture (fig. 1c, fig. 2d). An intravitreal bevacizumab (Avastin, $1.25 \mathrm{mg} / 0.05 \mathrm{ml}$; Genentech, Inc., San Francisco, Calif., USA) injection was administered, and 1 month later, the patient's BCVA of the left eye had improved to 20/32, and the CNV had shrunk (fig. 1d, fig. 2e). Also the IS/OS junction and the RPE had improved sufficiently and were able to be distinguished (fig. 2f).

\section{Discussion}

Choroidal rupture is a serious complication of nonpenetrating ocular trauma with a poor prognosis for a return to preinjury visual acuity. Traumatic choroidal ruptures can be divided into direct and indirect choroidal rupture according to the proximity of the original contusive injury site. Direct choroidal ruptures occur anteriorly, parallel to the ora serrata, and in proximity to the site of impact. Indirect choroidal ruptures occur posteriorly, apart from the site of the original impact, and originate from a countercoup injury against the 
Moon et al.: A Case of Expansion of Traumatic Choroidal Rupture with DelayedDeveloped Outer Retinal Changes

eyeball. Indirect choroidal rupture typically forms a crescent shape that is concentric with the edge of the optic disc, and $82 \%$ of indirect choroidal ruptures, like the present case, occur on the temporal side of the optic disc [6].

In this case, OCT examination revealed a rupture in both the RPE and Bruch's membrane in the macula, and distortions in the IS/OS junction and the ELM, yet the patient's BCVA was 16/20. Three weeks after the injury, an expansion of the RPE and the IS/OS junction, and an ELM disruption were observed, along with a sudden decrease in visual acuity. At 5 months after the injury, the size of the choroidal rupture had decreased.

Judging by the 3-week mark, the inflammatory response caused by the choroidal rupture may seem to cause expansion around the rupture area followed by contraction. However, considering the fact that the expansion had decreased 5 months after the injury, it is thought that the recovery resulted from the decreasing inflammatory response. It is uncertain whether the inflammation decreased due to the steroid treatment or natural resolution.

On the OCT examination 3 weeks after the injury, the distinction between the IS/OS junction and the RPE in the area adjacent to the ruptured retina was unclear. However after 5 months, the distinction was clearly observed. The abrupt vision decrease might have occurred due to the expansion of the choroidal rupture site along with changes in outer retina. And 5 months after the trauma, vision had improved as the outer retina regenerated. Francis and Freund [7] reported on photoreceptor reconstitution after intravitreal bevacizumab injection for treatment of secondary CNV. Another case report insists that ocular trauma destroys the photoreceptor OS and the RPE. However, after 7 days, the damaged photoreceptor OS had disappeared, and the IS had relocated to the RPE. Two weeks after the trauma, the OS had regenerated [3]. Studies have reported the derangement of the outer retina on OCT in patients with commotio retinae from trauma, and these studies share common evidence that a hyperreflective band in the photoreceptor layer was observed [8-11]. Mansour et al. [8] reported that a damaged area in commotio retinae is a junction of the photoreceptor OS and the RPE. Additionally, they assert that the photoreceptor OS of the outer retina stretch, while Müller cells keep the inner retina relatively stable [8]. A recent study on the natural history of the outer retina in spectral domain OCT after injury of a patient with commotio retinae reported that the IS/OS junction thickened and reflectivity increased, which resulted from a thinning of the semilucent layer between the IS/OS junction and the RPE [11]. Although the current case had similar OCT findings to those seen in commotio retinae, it is suspected the findings were posttraumatic inflammation change rather than commotio retinae. This is because commotio retinae was not identified in the ruptured retina in the fundus examination, and because of the unclear distinction between the IS/OS junction and the RPE adjacent to the ruptured retina 3 weeks after injury. The change in distinction after 5 months is presumed to have occurred as the inflammation decreased, which is similar to the improvement seen in the choroidal rupture.

After receiving steroid pulse therapy, the patient's visual acuity improved, and the size of the expanded choroidal rupture was diminished in OCT. In addition, the boundaries of the IS/OS junction and the RPE were clearly examined. However, it is uncertain whether the inflammation around the choroidal rupture had improved due to the steroid therapy or through natural resolution.

Five months after the injury, FAG and OCT examination revealed CNV, which was treated with an intravitreal bevacizumab injection. CNV shrinkage was detected 1 month after the injection. CNV occurs among $20 \%$ of patients with indirect choroidal rupture [5]. A secondary CNV developing after ocular contusion trauma may be related to a rupture of Bruch's membrane, inflammatory response, and abnormal production of vascular endotheli- 
Moon et al.: A Case of Expansion of Traumatic Choroidal Rupture with Delayed Developed Outer Retinal Changes

al growth factor. Many reports on the treatment of secondary CNV following choroidal rupture have been published [12-15], and the present case experienced an improvement following an intravitreal bevacizumab injection.

A PubMed search was performed without date restrictions (English-language results only) and with the use of the following terms: traumatic choroidal rupture and OCT. No report about the expansion of a choroidal rupture has been published to date. Furthermore, the reason for the expansion is not clear. Additional research is needed to discover the reason as well as the effect of steroid pulse therapy on the reduction of this expansion.

\section{Disclosure Statement}

None of the authors has a conflict of interest to disclose. No financial support was received for this work.

\section{References}

1 Youssri AI, Young LH: Closed-globe contusion injuries of the posterior segment. Int Ophthalmol Clin 2002;42:79-86.

2 Bellows JG: Observations on 300 consecutive cases of ocular war injuries. Am J Ophthalmol 1947;30:309323.

3 Hart JC, Natsikos VE, Raistrick ER, Doran RM: Indirect choroidal tears at the posterior pole: a fluorescein angiographic and perimetric study. Br J Ophthalmol 1980;64:59-67.

4 Wyszynski RE, Grossniklaus HE, Frank KE: Indirect choroidal rupture secondary to blunt ocular trauma. A review of eight eyes. Retina 1988;8:237-243.

5 Secretan M, Sickenberg M, Zografos L, Piguet B: Morphometric characteristics of traumatic choroidal ruptures associated with neovascularization. Retina 1998;18:62-66.

6 Aguilar JP, Green WR: Choroidal rupture. A histopathologic study of 47 cases. Retina 1984;4:269-275.

7 Francis JH, Freund KB: Photoreceptor reconstitution correlates with visual improvement after intravitreal bevacizumab treatment of choroidal neovascularization secondary to traumatic choroidal rupture. Retina 2011;31:422-424.

8 Mansour AM, Green WR, Hogge C: Histopathology of commotio retinae. Retina 1992;12:24-28.

9 Meyer CH, Rodrigues EB, Mennel S: Acute commotio retinae determined by cross-sectional optical coherence tomography. Eur J Ophthalmol 2003;13:816-818.

10 Sony P, Venkatesh P, Gadaginamath S, Garg SP: Optical coherence tomography findings in commotio retina. Clin Experiment Ophthalmol 2006;34:621-623.

11 Itakura H, Kishi S: Restored photoreceptor outer segment in commotio retinae. Ophthalmic Surg Lasers Imaging 2011;42:e29-e31.

12 Harissi-Dagher M, Sebag M, Gauthier D, Marcil G, Labelle P, Arbour JD: Photodynamic therapy in young patients with choroidal neovascularization following traumatic choroidal rupture. Am J Ophthalmol 2005;139:726-728.

13 Mennel S, Hausmann N, Meyer CH, Peter S: Photodynamic therapy and indocyanine green guided feeder vessel photocoagulation of choroidal neovascularization secondary to choroid rupture after blunt trauma. Graefes Arch Clin Exp Ophthalmol 2005;243:68-71.

14 Yadav NK, Bharghav M, Vasudha K, Shetty KB: Choroidal neovascular membrane complicating traumatic choroidal rupture managed by intravitreal bevacizumab. Eye (Lond) 2009;23:1872-1873.

15 Piermarocchi S, Benetti E, Fracasso G: Intravitreal bevacizumab for posttraumatic choroidal neovascularization in a child. J AAPOS 2011;15:314-316. 


\begin{tabular}{l|l}
\hline Case Rep Ophthalmol 2013;4:70-75 & \multicolumn{2}{l}{} \\
\hline DOI: 10.1159/000354197 & $\begin{array}{l}\text { @ 2013 S. Karger AG, Basel } \\
\text { www.karger.com/cop }\end{array}$ \\
\hline
\end{tabular}

Moon et al:: A Case of Expansion of Traumatic Choroidal Rupture with DelayedDeveloped Outer Retinal Changes
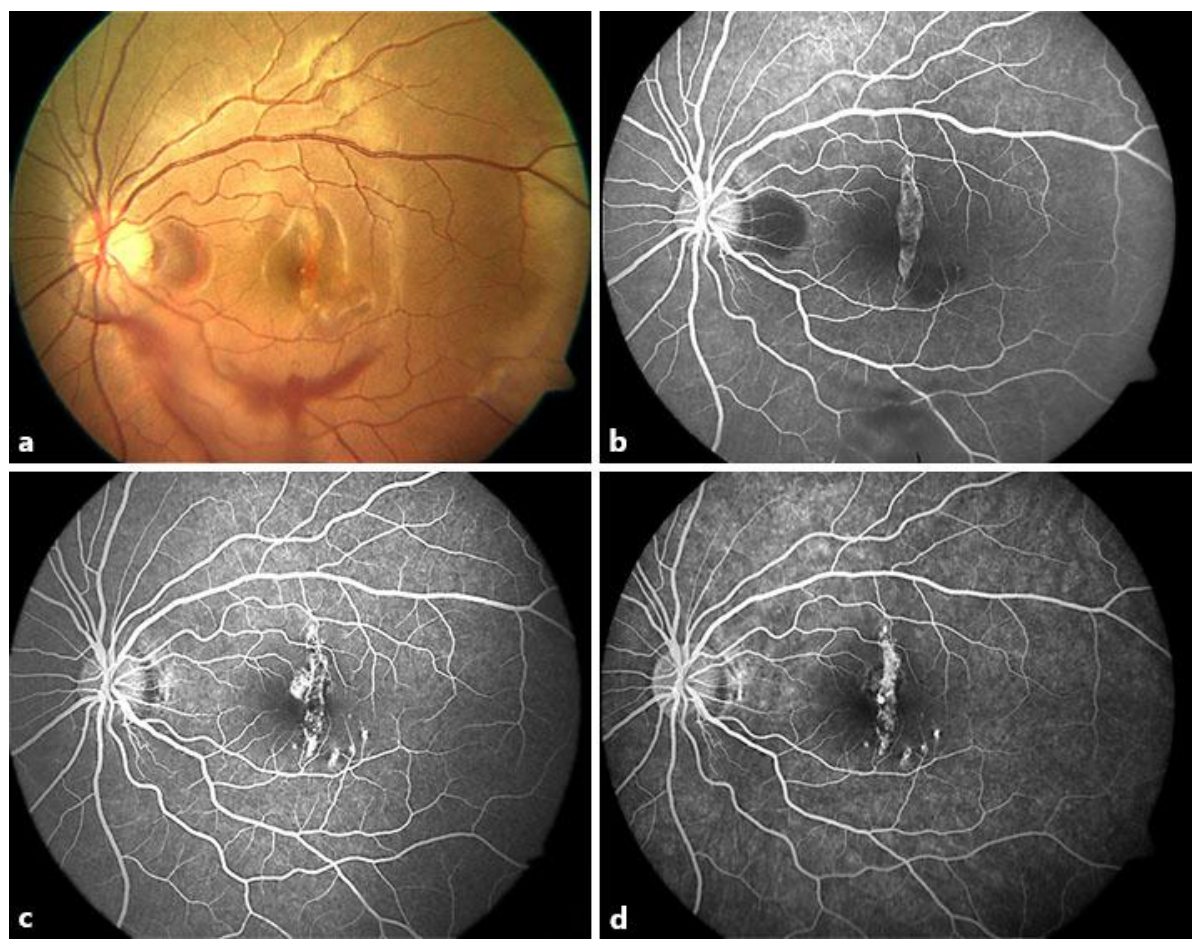

Fig. 1. Fundus photography and FAG findings. a At the first medical examination, choroidal rupture at the macula, subretinal hemorrhage at the posterior pole, and commotio retinae on the temporal side of the retina. b After 3 weeks, expansion of the choroidal rupture site presents as a window defect including the fovea. c Hyperfluorescence from CNV at the nasal edge of the rupture site after 5 months. $\mathbf{d}$ Staining of the choroidal rupture and scarred CNV 1 month after an intravitreal bevacizumab injection. 


\section{Case Reports in Ophthalmology}

\begin{tabular}{l|l}
\hline Case Rep Ophthalmol 2013;4:70-75 & \\
\hline DOI: 10.1159/000354197 & $\begin{array}{l}\text { @ 2013 S. Karger AG, Basel } \\
\text { www.karger.com/cop }\end{array}$ \\
\hline
\end{tabular}

Moon et al:: A Case of Expansion of Traumatic Choroidal Rupture with DelayedDeveloped Outer Retinal Changes

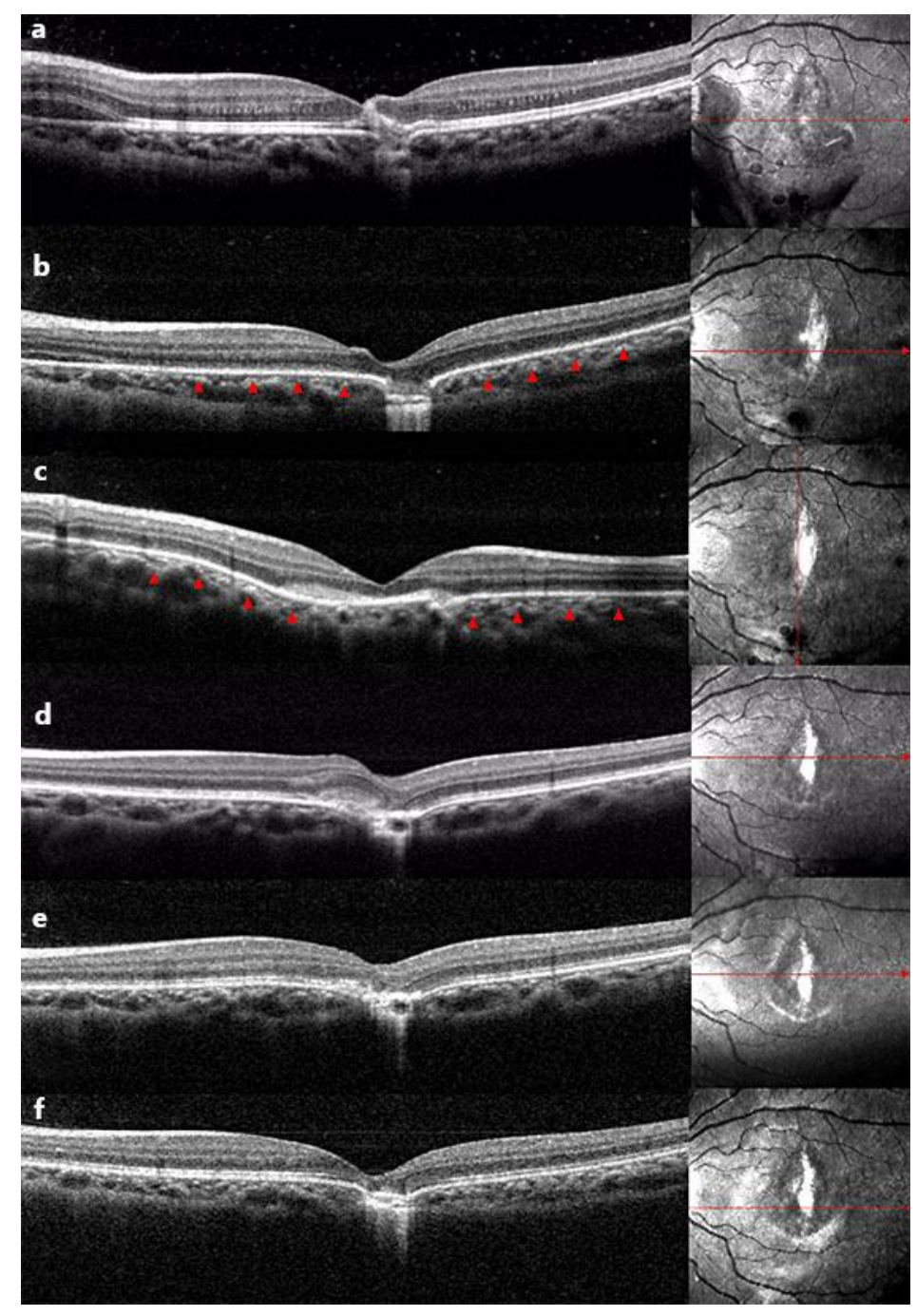

Fig. 2. OCT findings. a At the first medical examination, disruption of the retinal pigment epithelial layer with Bruch's membrane and distortion of the IS/OS junction and the ELM layer. b After 3 weeks, expansion of the choroidal rupture site and outer retinal changes (red arrowheads). c Vertical scan after 3 weeks, visible outer retinal changes (red arrowheads). $\mathbf{d}$ Newly developed CNV after 5 months. e Reduced CNV 1 month after an intravitreal bevacizumab injection. $f$ Recovery of the expanded choroidal rupture site and the outer retina 1 month after an intravitreal bevacizumab injection. 